\title{
FE-TOOL CODAC FOR AN EFFICIENT SIMULATION OF LOW-VELOCITY IMPACTS ON COMPOSITE SANDWICH STRUCTURES
}

\author{
Luise Kärger, Jens Baaran, Jan Teßmer \\ Institution \\ DLR, Institute of Composite Structures and Adaptive Systems \\ Lilienthalplatz 7, 38108 Braunschweig, Germany \\ luise.kaerger@dlr.de,jens.baaran@dlr.de,jan.tessmer@dlr.de
}

Keywords: Impact Simulation, Impact Damage, Sandwich Structures, Damage Mechanics, Finite Element Analysis.

\begin{abstract}
The finite element based damage tolerance tool, CODAC, has been developed for efficiently simulating the damage resistance of sandwich structures subjected to low-velocity impacts. The considered double shell structures consist of two thin composite face sheets separated by a lightweight core. While sandwich structures are very weight efficient and provide integrated damping and insulation, impact damage in sandwich structures can provoke a significant strength and stability reduction. Therefore, the objective of CODAC is to develop methodologies which reliably simulate impact events and accurately predict impact damage sizes.

Since frequent design loops require a quick analysis, efficient deformation and failure models are needed. To achieve a rapid and accurate stress analysis, a recently developed three-layered finite shell element is used. Failure analysis is based on a progressive damage mechanics approach: Damage initiation is detected by stress-based failure criteria. Material resistance is reduced by appropriate degradation models.

An experimental impact test program on honeycomb sandwich panels is used to validate the impact simulation of the FE-tool CODAC. Force-time histories and damage sizes are examined, and the influence of distinct failure models on the impact response is analyzed. Comparisons between impact tests and simulations showed that CODAC is capable of accurately and rapidly simulating impact events, which induce barely visible damage.
\end{abstract}




\section{INTRODUCTION}

Aircraft design calls for weight efficient shell constructions. Sandwich structures satisfy this demand by the combination of two thin, stiff face sheets and an intermediate lightweight core. Furthermore, the outer face sheet can act as an impact detector while the core provides damping and insulation. Thus, sandwich structures are increasingly aspired for application as fuselage and wing panels. However, impact damage in sandwich structures can provoke a significant strength and stability reduction. Therefore, the number of publications on impact behaviour of sandwich structures has recently increased. A number of experimental studies (e. g. [1][4]) were conducted to observe impact damage progression for different core and face sheet materials. The link between different stages of damage and changes in force-time histories was studied. It could be shown that invisible core crushing is the first damage mode which occurs during impact, it slightly lowers the slope of the force-time history. At higher energies, the contact force may reach a peak value, which was found to be an indicator for face sheet cracking.

To predict the impact response and the amount of impact damage, several simulation methodologies have been developed, [5]-[14]. Some of them are based on a simple analytical approach, which predicts damage initiation but does not describe damage progression, e.g. [5]-[8]. Furthermore, analytical models are restricted to special plate boundaries. By contrast, finite element simulations are flexible in terms of boundary conditions. Moreover, they account for progressive damage and non-linear material behaviour. Therefore, finite element approaches generally attain a more accurate description of the impact response. However, very fine FE models with a number of elements in thickness direction (e. g. [9]-[11]) require a large computational effort. On the other hand, much coarser models ([12]-[14]), may allow a more efficient simulation, but usually suffer losses in accuracy. For further information on publications about impact simulation see [15].

This paper presents the methodology of the finite element tool CODAC, whose main objective is a high efficiency in simulating impacts on sandwich structures, chapter 2. In section 2.1 a three-layered finite shell element is introduced to model the deformation and stress behaviour of sandwich structures. Instruments of the transient impact analysis are explained in section 2.2. The main focus of this paper is the validation of impact simulation results, chapter 3. where an impact test program is used for comparison, section 3.1. Simulation results in terms of force-time histories and core damage sizes are presented and evaluated for different degradation models in sections 3.2 to 3.4 . Finally, information is given about the computational effort of impact simulations with CODAC.

\section{DAMAGE TOLERANCE TOOL CODAC}

The DLR in-house tool CODAC (Composite Damage Tolerance Analysis Code) is a fast finite element tool, which is used in the design process to efficiently simulate the low-velocity impact behaviour of composite panels and to compute the residual strength, [16], [17]. CODAC was originally designed for monolithic CFRP structures. To meet the increasing interest of the aircraft inductry in double shell structures, it is presently enhanced for sandwich panels.

\subsection{Finite sandwich elements}

To be used in the design process, the impact simulation methodologies need to be fast and at the same time sufficiently accurate. Since a reliable deformation and stress prediction is an essential precondition for an accurate failure analysis, a suitable finite element is needed, which 
predicts in-plane stresses as well as transverse shear and normal stresses precisely enough. Consequently, a suitable finite element should be capable of efficiently modelling the characteristic deformation behaviour of double shell structures: Large shear strains may occur in the soft core, whereas the cross-sections of the skins remain almost orthogonal to the in-plane direction. By considering and evaluating a couple of finite element concepts, two new sandwich elements have been developed.

The first sandwich element, called S89 and proposed by Kärger et al. [18], is a three-layered shell element, which is based on a plane stress assumption. It is described by eight nodes and serendipity form functions. The displacement approach in thickness direction is developed by using the first-order shear deformation theory (FSDT) for each of the three layers (core and two skins), coupled by displacement continuity conditions at the interfaces. Consequently, each node incloses nine degrees of freedom. In order to improve transverse stiffness and stresses, an enhancement of the Extended 2D Method by Rolfes and Rohwer [19] was developed.

It has turned out, however, that under concentrated out-of-plane loads element S89 lacks appropriate accuracy of stress results. Therefore, an improved finite element formulation was developed by Wetzel et al. [20], which accounts for the full 3D sress state. Due to its 15 degrees of freedom per node, this element was called S815. Analogous to the former element S89, it is an isoparametric eight-node shell element and the in-plane displacements are described by a layer-wise linear approach in thickness direction. As a distinction to element S89 the compressibility in thickness direction is included in the kinematical approach. This addition is made to better account for the local transverse strains due to concentrated impact loading in connection with a soft core material. In order to avoid Poisson thickness locking, a parabolic distribution was chosen for the out-of-plane displacement. Hence, the kinematics of each of the three layers $L$ is described by

$$
\left[\begin{array}{c}
u_{L}(x, y, z) \\
v_{L}(x, y, z) \\
w_{L}(x, y, z)
\end{array}\right]=\left[\begin{array}{c}
u_{L}^{0}(x, y) \\
v_{L}^{0}(x, y) \\
w_{L}^{0}(x, y)
\end{array}\right]+z_{L}\left[\begin{array}{c}
\psi_{x L}(x, y) \\
\psi_{y L}(x, y) \\
\psi_{z L}(x, y)
\end{array}\right]+\left(z_{L}\right)^{2}\left[\begin{array}{c}
0 \\
0 \\
\varphi_{z L}(x, y)
\end{array}\right]
$$

cf. Figure 1. Using the compatibility conditions at the layer interfaces, the deformation state at a node can be described by three transformations $\left(u_{0}, v_{0}, w_{0}\right)$, two rotations per layer $\left(\psi_{x L}\right.$, $\left.\psi_{y L}\right)$ and two additional degrees of freedom per layer for the displacement in thickness direction $\left(\psi_{z L}, \varphi_{y L}\right)$. Due to its transverse compressibility, element $\mathrm{S} 815$ has an additional advantage over element S89: It is capable of modelling impacts on completely supported plates, which is a boundary condition that is often used in experimental impact tests on sandwich plates. Additionally, the feasibility of complete support is the main reason for locating the shell reference plane in the bottom face sheet, as can be seen in Figure 1 .

Since stress-based failure criteria are used for failure detection, the accuracy of stress computation is very important. Of course, the full 3D-material law can be used to evaluate stresses; and indeed, the material law provides very good results for in-plane stresses. However, the quadratic approach of the out-of-plane displacements is not flexible enough to give satisfying results also for transverse stresses. Much better transverse shear stresses are obtained by utilizing the equilibrium conditions of the in-plane forces

$$
\left[\begin{array}{c}
\tau_{x z L} \\
\tau_{y z L}
\end{array}\right]=-\int_{\zeta=-\frac{h_{1}}{2}}^{z}\left[\begin{array}{l}
\sigma_{x x, x}^{(k)}+\tau_{x y, y}^{(k)} \\
\sigma_{y y, y}^{(k)}+\tau_{x y, x}^{(k)}
\end{array}\right] d \zeta
$$




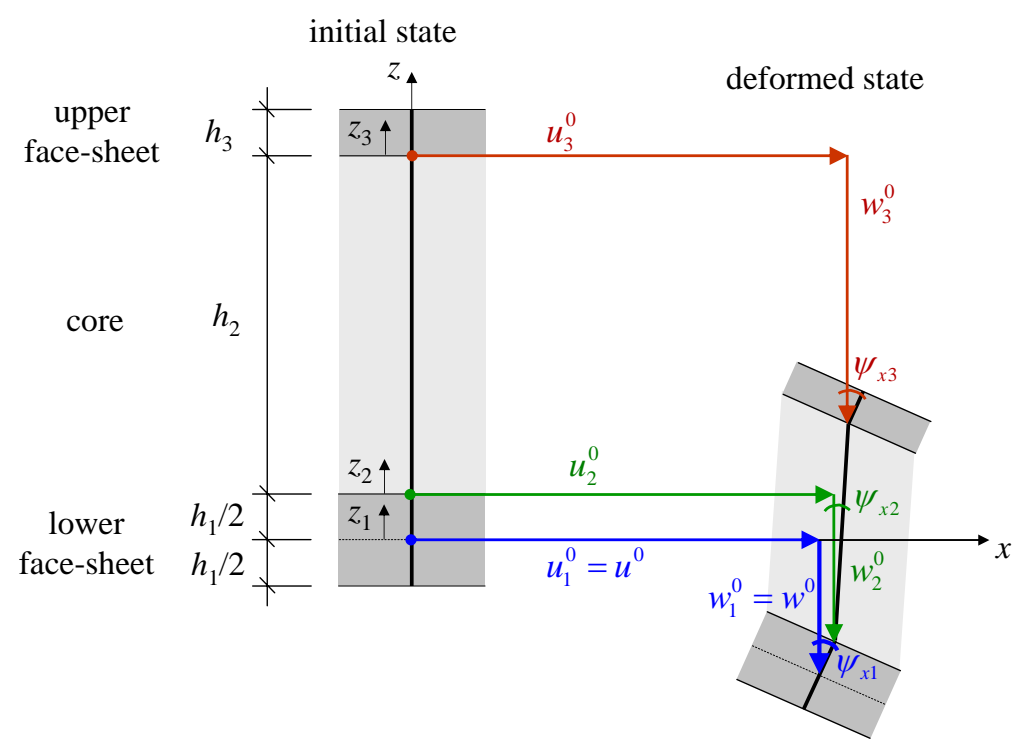

Figure 1: Kinematic approach of element S815

In order to avoid the necessity of second derivatives of the shape function, the equilibrium equation is modified in a way that the transverse shear stresses can be described as a function of the transverse shear forces,

$$
\begin{aligned}
\boldsymbol{\tau}_{z 1}(x, y, z) & =\mathbf{f}_{1}(z) \cdot \mathbf{R}(x, y) \\
\boldsymbol{\tau}_{z L}(x, y, z) & =\mathbf{f}_{L}(z) \cdot \mathbf{R}(x, y)+\tau_{z(L-1)}\left(z_{L 0}\right) \quad(L=2,3) .
\end{aligned}
$$

This procedure builds on the above mentioned enhancement of the Extended 2D Method [19] for three-layered shell elements, which was proposed by Kärger et al. [18].

The suchlike improved transverse shear stresses have the advantage of being linear in inplane direction. Therefore, they can be differentiated and used to calculate transverse normal stresses by solving the equilibrium equation in thickness direction,

$$
\sigma_{z z L}=-\int_{\zeta=-\frac{h_{1}}{2}}^{z}\left(\tau_{x z, x}+\tau_{y z, y}\right) d \zeta+q_{z 0},
$$

where $q_{z 0}$ are the external loads in thickness direction, cf. Rolfes et al. [21]. Since here the second derivatives of the shape function are unavoidable, the transverse normal stresses are element-wise constant in in-plane direction and, therefore, the optimal stress point is in the element centre rather than in the four Gauß points.

Computational tests, where the analytical solution by Pagano [22], [23] was used for comparison, have shown that the equilibrium method provides very good transverse stresses: Transverse shear stresses were always better than those from material law. For plates with smoothly distributed loads, transverse normal stresses could also be improved. However, plates with concentrated loads, like impacts, caused some more difficulties: Because of the extreme local stress concentration, oscillations in the transverse normal stresses cannot be completely avoided, neither by the equilibrium approach nor by the material law. In order to minimise these oscillations, on one hand, the impact force is modelled by a parabolically distributed surface load, whose area grows consistenly with the Hertzian contact area. On the other hand, for stress-based failure analysis a combination of both methods is used, which applies in each case the more critical 
value of the two stress results. Therewith, the transverse normal stress computation also give reliable and conservative results.

\subsection{FE-modelling and transient impact analysis}

An impact simulation technique of sandwich structures, which makes use of the new sandwich elements S89 and S815, was first published by Kärger et al. [15]. Fig. 2 exemplifies the finite element modelling used in CODAC for impact analysis. The impacted area is meshed a lot finer than the outer parts of the panel to be able to correctly predict incremental damage growth near the stress concentration while keeping the computational effort as low as possible. The Figure shows one of the meshes, which was used to simulate the impact tests introduced

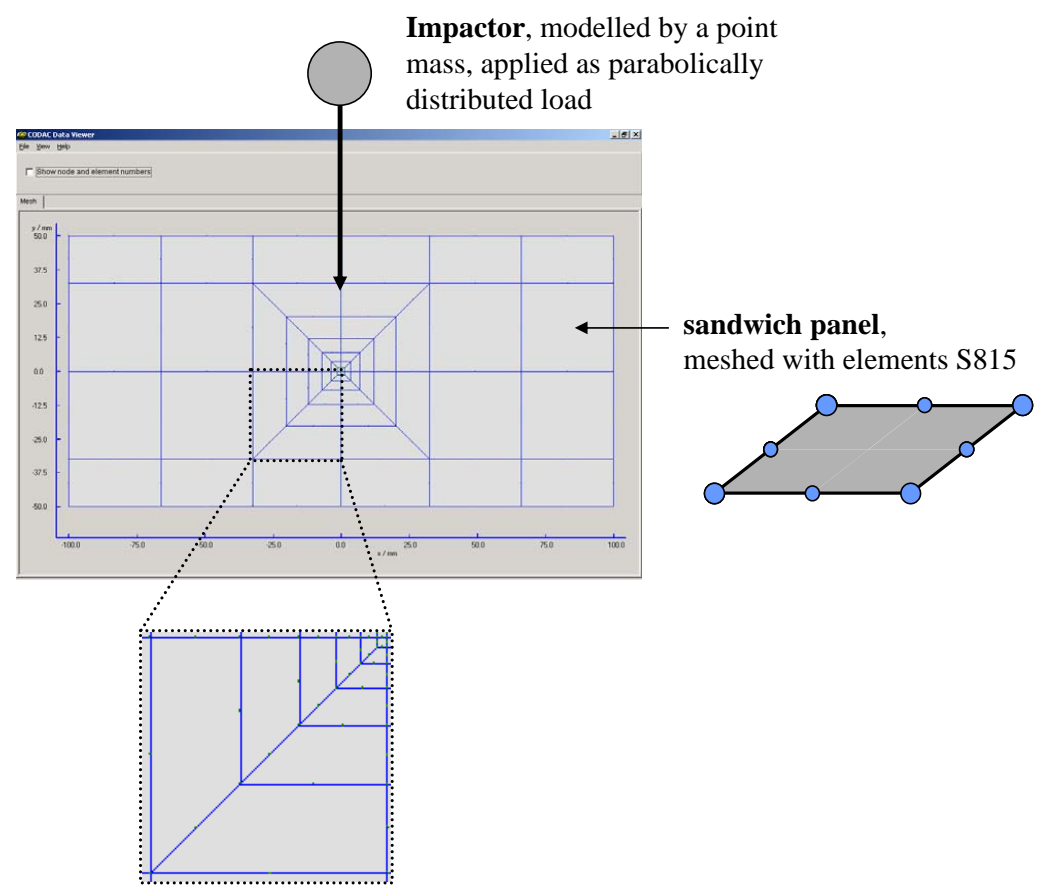

Figure 2: FE-modelling for impact analysis.

in chapter 3. If the lay-up of the laminate is symmetric, it is sufficient to model a quarter of the panel. Furthermore, computational tests have shown that only a bounded inner region of the plate is affected by the impact load. Therefore, in the simulations of chapter 3 it was sufficient to model a smaller, quadratic part, shown by the zoom in Fig 2. It might appear that the sandwich plate is meshed rather coarsely. However, the here introduced mesh turned out to be precise enough to achieve good results, as demonstrated in section 3.2 .

The impactor is modelled by a point mass. The contact force between impactor and plate is distributed parabolically. The magnitude of the impact load as well as the expanse of the loaded area is calculated via the Hertzian contact law. For simulating the dynamic process, CODAC uses the implicit Newmark time integration scheme. Comparative studies have shown that the explicit central difference method requires extremely small time steps to achieve stability. Therefore, implicit time integration was found to be more efficient for the demands of the design tool CODAC. 


\section{IMPACT SIMULATION RESULTS}

\subsection{Impact tests used for validation}

The impact tests, which are used in the following to validate the impact simulation techniques, were conducted at the department ILR at Dresden University of Technology [24]. Test results in the form of force-time histories, damage photographs and ultrasonic scans were provided by courtesy of ILR Dresden. Figure 3 shows the impact test facilities of ILR. The sand-

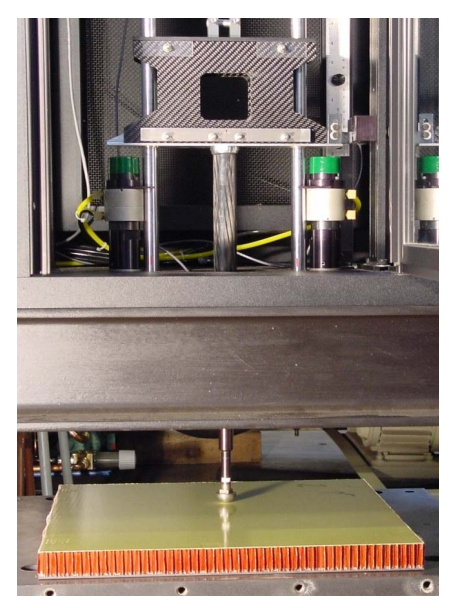

Figure 3: Impact facilities at ILR Dresden.

wich panels were completely supported and impacted with a steel hemisphere $(\oslash 25.4 \mathrm{~mm}$, $1.10 \mathrm{~kg}$ ) and energies between $1 \mathrm{~J}$ and 15J. The panels consisted of NOMEX honeycomb 4.8-48 core of 28mm thickness and Cytec 977-2/HTA face sheets. Since the top face sheet shall act as impact detector, while the main inplane loads are carried by the bottom face sheet, the sandwich is asymmetric and the impacted face sheet is very thin with three plies and a nominal thickness of only $0.633 \mathrm{~mm}$.

Force-time and displacement-time histories (Fig. 4) are important experimental results for impact tool validation. Whereas the force-time curve of the $1 \mathrm{~J}$ impact is almost sine-like, at the $8 \mathrm{~J}$ and $15 \mathrm{~J}$ impacts a sharp bend occurs at about $1 \mathrm{kN}$, which coincides with an indentation of about $3 \mathrm{~mm}$. This sharp bend indicates a massive damage, which can be identified as the begin of face sheet cracking. Another, but slight bend can be seen in all force-time curves at a very low energy-level. This indicates a stiffness reduction, which is caused by core crushing.

A further indication for failure behaviour during impact is given by damage images. In Figures 5 and 6 the middle, ultrasonic images show the condition of the top face sheet, where black areas are actual face sheet damages and the grey areas show the interface between skin and core. At $1 \mathrm{~J}$ impact, where a contact force of $1 \mathrm{kN}$ is not reached, the top face sheet does not experience visible damage (Fig. 5). On the other hand, at $8 \mathrm{~J}$ the skin is considerably affected (Fig. 6). Contrary to the first ultrasonic scan, the right ultrasonic scan indicates core failure, since here the complete sandwich, inclusive core, is scanned. It can be seen that considerable core failure occurs already at $1 \mathrm{~J}$.

\subsection{Impact simulation without failure analysis}

To validate the pure deformation analysis during impact, which is performed by the new sandwich elements S815, the Hertzian contact law and the Newmark time stepping scheme 

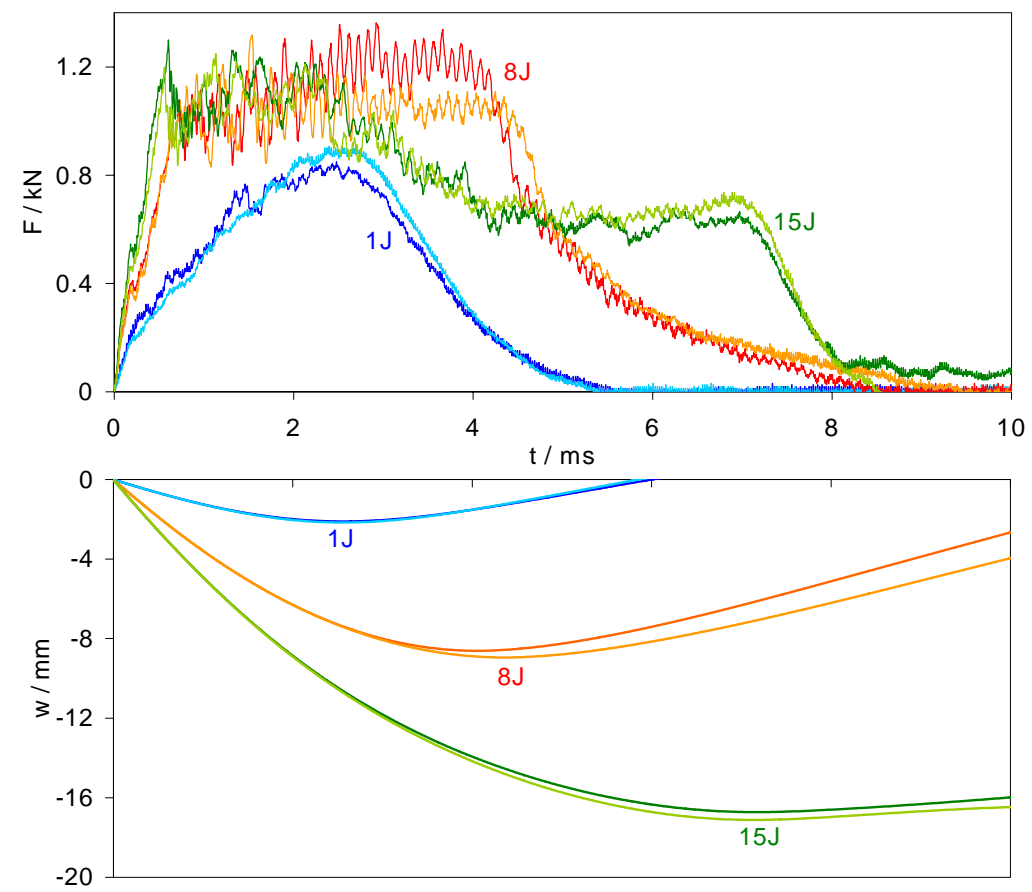

Figure 4: Force-time and displacement-time histories from impact tests at ILR Dresden.
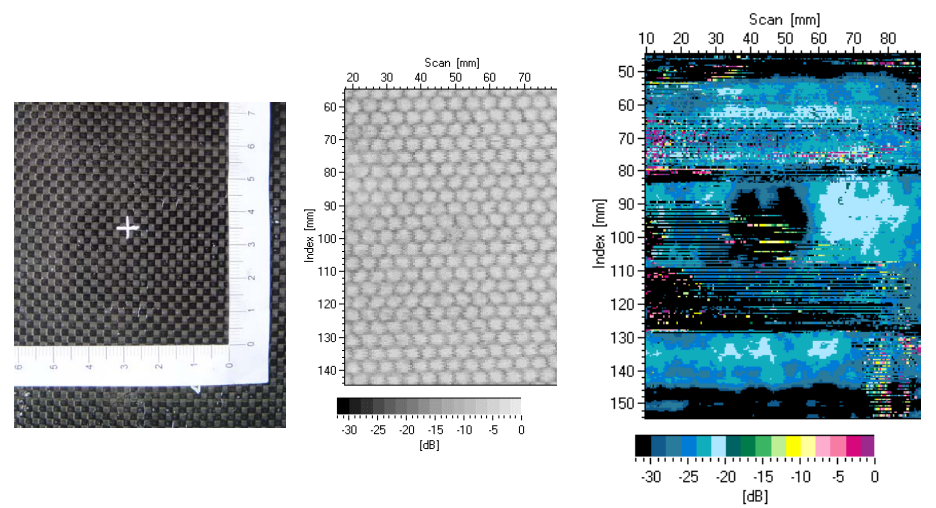

Figure 5: Damage images from ILR Dresden: photograph and US scans (echoes of top and bottom skin), 1J impact.
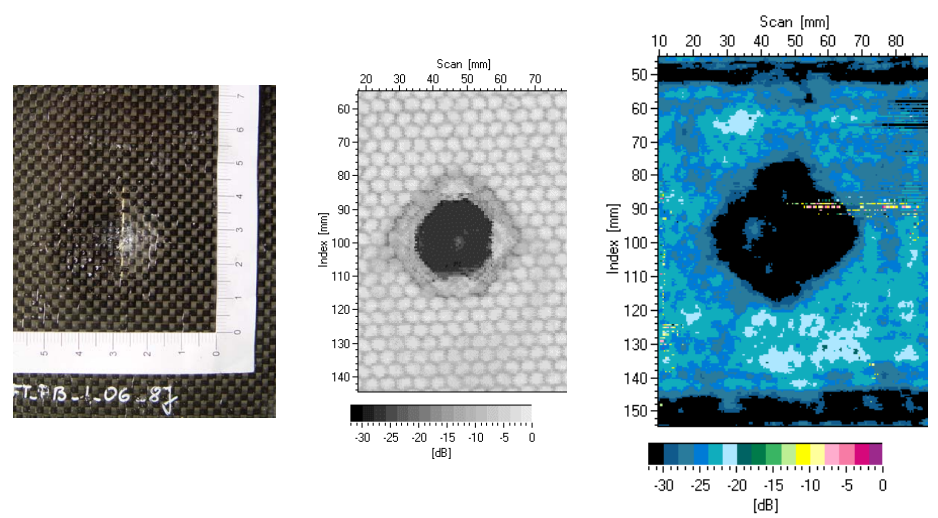

Figure 6: Damage images from ILR Dresden: photograph and US scans (echoes of top and bottom skin), 8J impact.

(section 2.2), the simulation is first carried out without any material degradation. Analysing the impact response without degradation means that all stiffness components of the sandwich 
plate remain constant during the whole impact analysis. Fig. 7 shows the force-time histories for a $1 \mathrm{~J}$ impact. The two red experimental results show only little scatter. The four FE analysis curves demonstrate differences for different meshing and different loading: For the blue curves the load is modelled by a single nodal load, for the green curves the load is parabolically distributed. It can be seen that applying a single nodal load results in a mesh dependent response, i.e. the modelled plate is increasingly compliant for finer meshes (curve of the mesh with 38 elements is lower than the curve of the mesh with 14 elements). On the other hand, the two green curves overlap almost completely and indicate mesh independent results. Furthermore, the initial slopes, which represent the stiffness of the intact material, agree with the slopes of the experimental curves very well. Therefore, in all following simulations, the impact load is parabolically distributed and the coarser mesh is used, since it is precise enough to achieve mesh independent results.

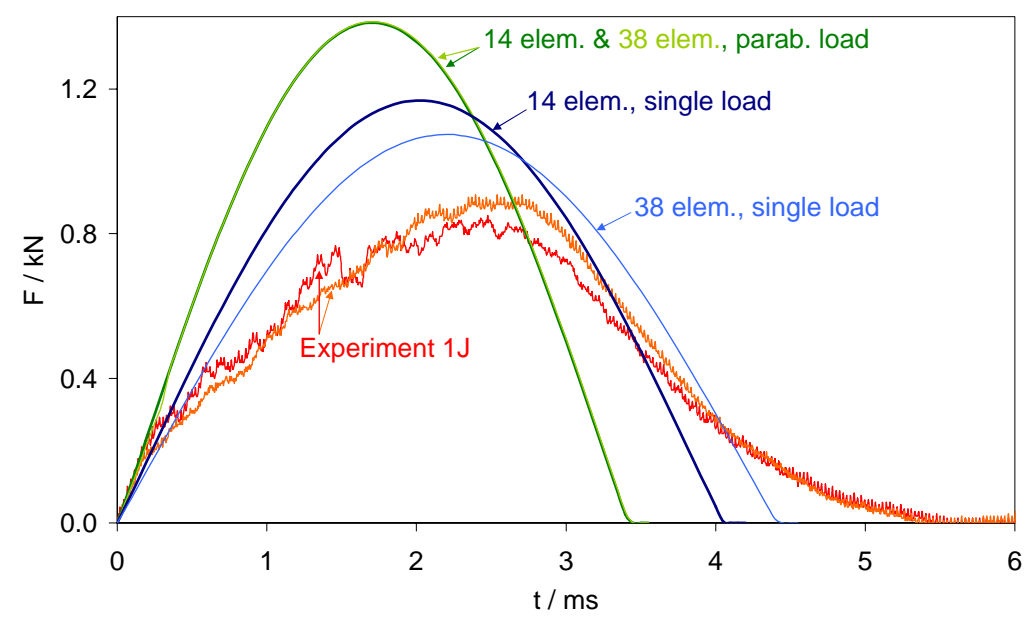

Figure 7: Without degradation: Force-time histories of a 1J impact.

The computed curves leave the experimental curves already at a small contact force of about $0.25 \mathrm{kN}$. At this point, the core starts to crush and, consequently, the stiffness of the plate decreases. In order to correctly describe the further impact response, core failure needs to be detected and subsequently core material needs to be degraded.

\subsection{Impact simulation including a core failure model}

The core fails due to combined shear and compression. Consequently, for detecting failure initiation, the failure criterion by Besant et al. [13] is used, which includes both, transverse normal and transverse shear stresses,

$$
\left(\frac{\sigma_{z z}}{\sigma_{\mathrm{cu}}}\right)^{n}+\left(\frac{\tau_{x z}}{\tau_{\mathrm{lu}}}\right)^{n}+\left(\frac{\tau_{y z}}{\tau_{\mathrm{tu}}}\right)^{n} \geq 1 .
$$

In Besant's combined shear-compression-tests, an exponent of $n=1.5$ produced the best fit with experimental results. Therefore, in the impact analysis of CODAC, $n=1.5$ is defined as default value. However, numerical test runs with exponents between 1.0 and 2.0 did not show a remarkable influence on the force-time history or on final impact damage.

Fig. 8 shows force-time histories of a $1 \mathrm{~J}$ impact. For two computed curves a core failure is taken into account. In both cases, onset of core damage is correctly detected, which demonstrates the capability of the failure criterion. 


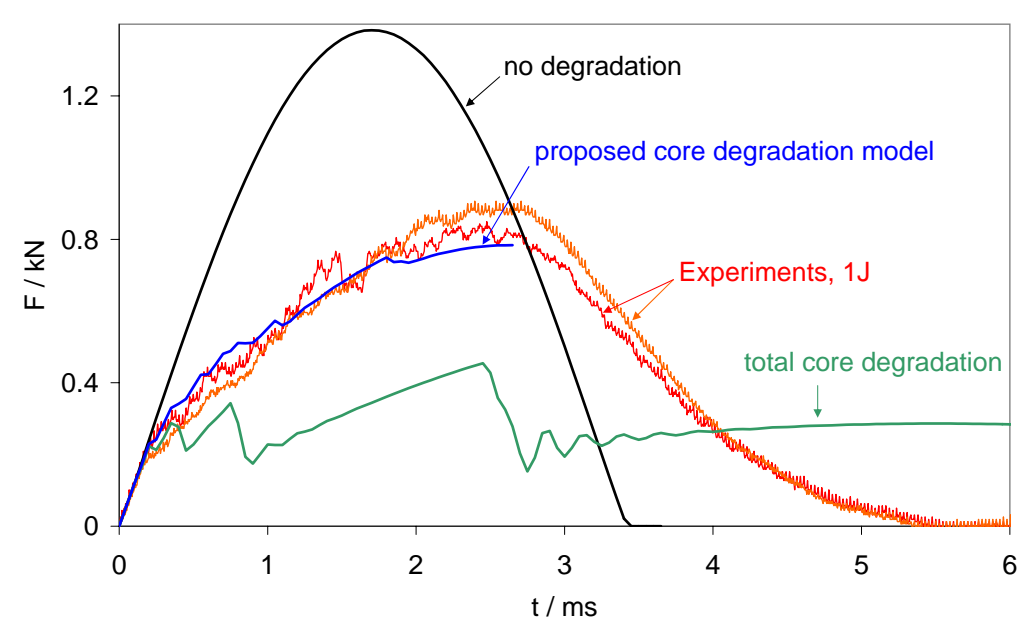

Figure 8: With core degradation: Force-time histories of a $1 \mathrm{~J}$ impact.

If the material of all damaged core regions is totally degraded, i.e. all stiffness components are set to a value close to zero, the green curve in Fig. 8 arises. Evidently, such a total core degradation models the damaged core too soft and results in an underestimation of the contact force. Furthermore, this computation heavily overestimates the damaged area: In the second picture of Fig. 9 the area with blue crosses, which shows the computed damage area, is much larger than the red circle, which displays the border line of the experimental damage area.


Figure 9: With core degradation: Core damage of a $1 \mathrm{~J}$ impact.

Consequently, a more sophisticated degradation model is needed, which does not just treat the damaged core as non-existing but really looks at its material behaviour. Therefore, the stress-strain-path of the core material is investigated and, subsequently, implemented into the core failure model. Exemplarily, Fig. 10 shows force-displacement curves of compression tests, which were carried out at ILR Dresden to characterise the material behaviour of the studied honeycomb core. It can be seen that after reaching ultimate strength, the stresses do not drop to zero as assumed before, but the core crushes at a certain stress level. Due to this remaining crush strength, the damaged core absorbes energy, which needs to be regarded in the Principle of Virtual Work. By representing this additional energy in the finite element stiffness relation between nodal displacements and nodal forces, an additional inner nodal force arises, which operates in the opposite direction of the outer contact force and, therewith, provides a considerable residual resistance of the damaged core. Furthermore, after reaching the crush strength level, the stresses slightly increase. Therefore, the corresponding stiffness component must not be set to zero, but reduced according to the reduced slope of the material's stress-strain path. Summarising, the failure model for core compression, which arises by taking both, the residual strength and residual stiffness into account, is the step-wise linear function shown by the black curve in Fig. 10 . 


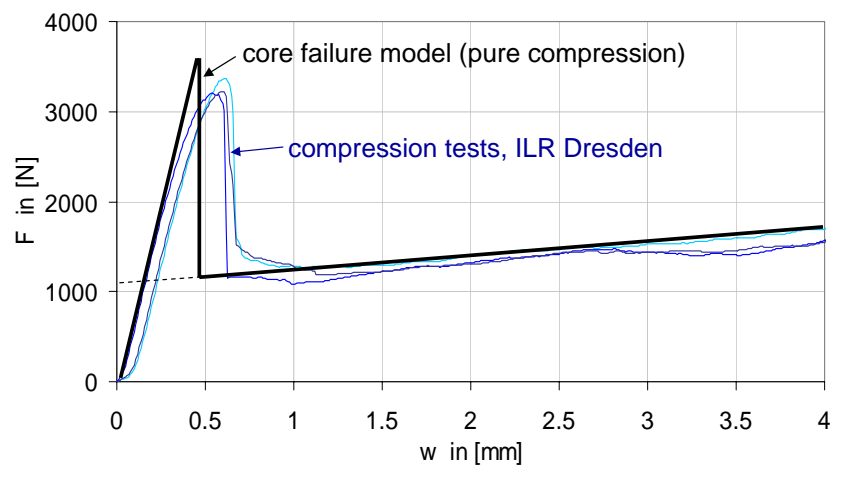

Figure 10: Force-displacement curve of core compression.

At shear loading, a force-displacement relation similar to the one in Fig. 10 can be observed. Since the true failure behaviour of the core is a combination of transverse compression and transverse shear, an average remaining resistance has to be assumed. Under consideration of typical strain values and the associated amount of absorbed energy, crush strength and crush stiffness values can be approximated (see Table 1 for the values used in this study). In order to avoid numerical instability due to an ill-conditioned finite element system of equations, the remaining shear resistance is modeled by an average crush stiffness combined with zero crush strength. For more detailed information on CODAC's core damage modelling see [15].

Table 1: Factors for crush strength and crush stiffness of damaged core

\begin{tabular}{lccc}
\hline & $\begin{array}{c}\text { Compression } \\
(i=3)\end{array}$ & $\begin{array}{c}\text { Shear xz } \\
(i=5)\end{array}$ & $\begin{array}{c}\text { Shear yz } \\
(i=4)\end{array}$ \\
\hline$\sigma_{i, \text { crush }} / \sigma_{i, \text { ult }}$ & 0.3 & 0 & 0 \\
\hline$C_{i i, \text { crush }} / C_{i i}$ & 0.02 & 0.3 & 0.4 \\
\hline
\end{tabular}

By applying the proposed core degradation model during the impact simulation, the experimental results are approximated much better than by using the above introduced total degradation model, cf. blue curve in Fig. 8 and right picture in Fig. 9. The force-time curve is displayed here only up to maximum deflection, because the unloading stress-strain-path of the plastically deforming core is not implemented yet. It can be concluded that for impacts without visible face sheet damage, the force-time history curves are very well described and a quite accurate damage area is computed.

However, for an increasing impact energy, contact forces larger than $1 \mathrm{kN}$ and remarkable skin damages are expected. Fig. 11 shows the force-time histories of an $8 \mathrm{~J}$ impact: In accordance with the $1 \mathrm{~J}$ impact, the initial stiffness is well approximated and the first core damage at about $0.4 \mathrm{kN}$ is correctly detected. Furthermore, by considering the proposed core degradation model, the remaining stiffness of the damaged core is accurately modeled.

However, at about $1.0 \mathrm{kN}$ the upper face sheet experiences a substantial damage. Thus, face sheet degradation needs to be considered to be able to keep on following the experimental force-time curve.

\subsection{Failure and indentation behaviour of face sheets}

In CODAC, several approved stress-based failure criteria and stiffness degradation models for fibre breakage, matrix cracking and delamination are available. Parametric studies were 




Figure 11: With core degradation: Force-time histories of an $8 \mathrm{~J}$ impact.

carried out to analyse the influence of skin failure criteria and skin degradation models on the impact response of the sandwich panels.

The studies showed that fibre breakage is the most relevant failure mode, while degradation due to matrix cracking and especially due to delamination has little influence on force time histories. Even though there might actually occur some matrix cracking or delamination before $1.0 \mathrm{kN}$, these minor damages are not responsible for a remarkable stiffness reduction during impact. Consequently, for detecting the onset of remarkable skin damage, stress-based failure criteria for fibre breakage were analysed: the maximum stress criterion, Hashin fibre failure criterion [25] and Chang-Chang fibre failure criterion [26]). It was found that all three criteria predict fibre failure too early, which means that either stress-based criteria are not appropriate for skin failure detection, or that in-plane stresses are larger than in reality. The first consideration follows the idea that at heavily concentrated stresses, which undoubtedly occur under impact loads, the application of energy-based criteria is more suitable than stress-based criteria. The second thought assumes a shortcoming of the displacement model at large deformations.

If a thin sandwich skin is impacted with high energies, and if the core is comparatively soft, the skin experiences large deformations. In order to model the deformation behaviour still correctly, the strain-displacement relation has to contain non-linear terms, which regard the membran effect of large deflections. A suggestion considering membrane forces was made by Olsson [27], who proposed an analytical membrane solution in addition to the plate solution for sandwich skins and observed its importance at higher indentations. Consequently, the sandwich element S815, which was introduced in section 2.1, was enhanced by non-linear strain-displacement terms to account for large deflections.

To analyse the effects of non-linear strain terms, comparative computational tests were conducted: The above introduced sandwich panel was loaded by static loads, which represented the impact load. In Fig. 12, the non-linear results for skin deflection and skin in-plane stresses are compared to linear results. It can be seen that the effect of non-linear strain terms is already remarkable at deflections of about $3 \mathrm{~mm}$ (for comparison: max. deflection at $8 \mathrm{~J}$ impact were $\approx 9 \mathrm{~mm}$, max. deflection at $1 \mathrm{~J}$ impact were $\approx 2 \mathrm{~mm}$, cf. Fig. 4). The reason why the static load $\mathrm{F}$ in Fig. 12 is much larger than the impact forces in Figures 4,8 and 11 is given by the fact that here core degradation is not regarded. Nevertheless, for same deflections the effect of nonlinear terms is similarly valid with a degraded core, since the non-linearity mainly depends on the displacement state of the skin and not on the stress state in the core. 

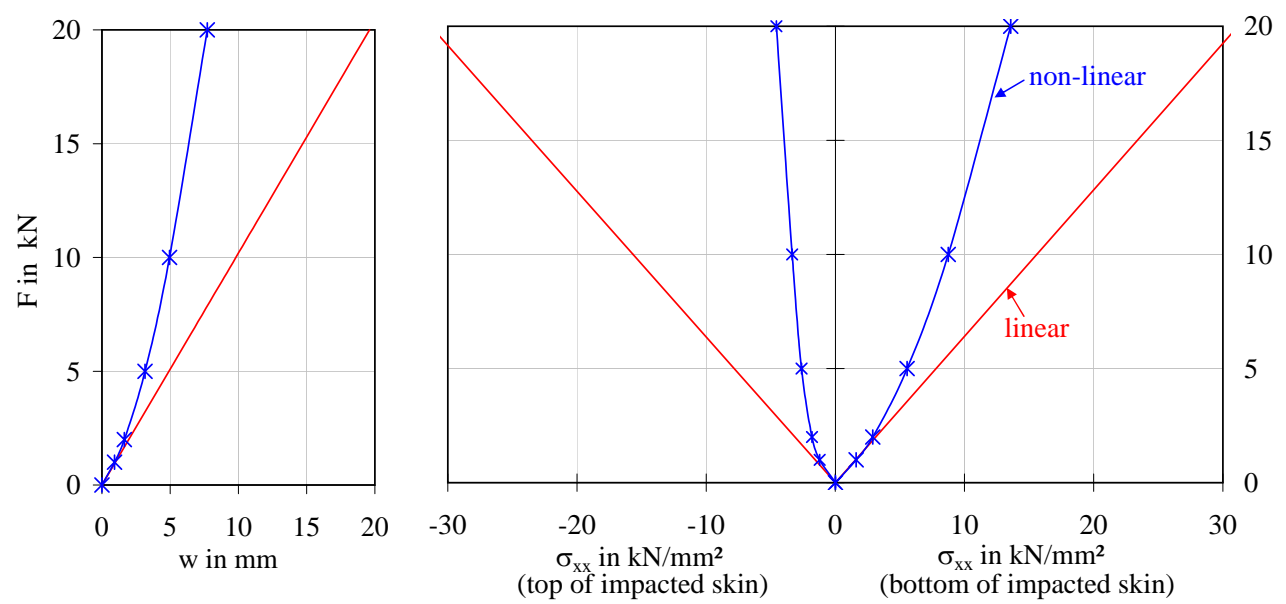

Figure 12: Geometrical non-linearity: Deflection and in-plane stresses.

Recapitulating, at impact simulations with high energies, considering the non-linear strain terms of the impacted face sheet will result in smaller indentations and in smaller in-plane stresses. Consequently, fibre breakage will be detected later than without geometrical nonlinearity and the impact simulation will come closer to experimental results. However, including the geometrically non-linear element S815 in the impact analysis and modeling the fracture behavior of the impacted skin is the subject of currently ongoing investigations.

\subsection{Computational effort}

Depending on the impact energy level and the extent of degradation, a CODAC simulation of a sandwich panel with the introduced mesh takes between 10sec and 1:30min on a personal computer with $2.3 \mathrm{GHz}$. Due to its rapidity, the simulation is applicable for design processes.

\section{CONCLUSION}

The finite element tool CODAC and its methodology to efficiently simulate impacts on sandwich structures was presented. A recently developed three-layered finite shell element allows an accurate and efficient reproduction of stress and deformation behavior during impacts on sandwich structures. The simulation was found to be mesh independent by parabolically distributing the contact force according to the Hertzian contact area. Comparisons between impact tests and simulations showed that the stiffness of the undamaged sandwich plate is well modeled.

An appropriate stress-based failure criteria for core crushing is capable of accurately detecting damage initiation. The material behavior of the damaged core is suitably described by a refined core degradation model. As a consequence, core damage sizes due to impact are well predicted. Moreover, impact events of low energy, which induce barely visible damage, can be accurately and rapidly simulated.

At higher impact energies, the impacted face sheet experiences large deflection. It could be shown that before reaching a skin damaging load, it is essential to account for geometrical nonlinearity in order to compute displacements and stresses correctly. Consequently, for predicting the initiation of skin failure, non-linear strain terms have been included in the top skin layer of the proposed finite shell element. The implementation of the geometrically non-linear element in the impact analysis is currently investigated. 


\section{ACKNOWLEDGMENT}

The presented work is funded by the National Aeronautical Research Program. The authors are grateful to the Department of Aerospace Technology at Dresden University (ILR Dresden) for conducting the test program and providing the experimental results. Furthermore, the help of our student assistant Markus Kintscher by executing the computational test program is gratefully acknowledged.

\section{REFERENCES}

[1] E.J. Herup and A.N. Palazotto, Low-velocity impact damage initiation in graphite/epoxy/Nomex honeycomb-sandwich plates. Composite Science and Technology, 57, 1581-1598, 1997.

[2] J. Gustin, A. Joneson, M. Mahinfalah, J. Stone, Low velocity impact of combination Kevlar/carbon fibre sandwich composites. Composite Structures, 69, 396-406, 2005.

[3] J.P. Dear, H. Lee, S.A. Brown, Impact damage processes in composite sheet and sandwich honeycomb materials. International Journal of Impact Engineering, 32, 130-154, 2005.

[4] P.M. Schubel, J.-J. Luo, I.M. Daniel, Low velocity impact behavior of composite sandwich panels. Composites Part A, 36, 1389-1396, 2005.

[5] R. Olsson, Prediction of impact damage in sandwich panels. Proc. Third Int. Conf. on Sandwich Construction, 659-668, 1996.

[6] A. Petras, M.P.F. Sutcliffe, Indentation failure analysis of sandwich beams. Composite Structures, 50, 311-318, 2000.

[7] M.S. Hoo Fatt, K.S. Park, Dynamic models for low-velocity impact damage of composite sandwich panels - Part B: Damage initiation. Composite Structures, 52, 353-364, 2001.

[8] R. Olsson, Engineering method for prediction of impact response and damage in sandwich panels. Journal of Sandwich Structures and Materials, 4, 3-29, 2002.

[9] F. Hähnel, K. Wolf, Numerical simulation of CFRP honeycomb sandwich subjected to low-velocity impact. Proc. Fifth Int. Conf. on Sandwich Construction, 2000.

[10] M. Meo, R. Vignjevic, G. Marengo, The response of honeycomb sandwich panels under low-velocity impact loading. International Journal of Mechanical Sciences, 47, 13011325, 2005.

[11] M.Q. Nguyen, S.S. Jacombs, R.S. Thomson, D. Hachenberg, M.L. Scott, Simulation of impact on sandwich structures. Composite Structures, 67, 217-227, 2005.

[12] A.N. Palazotto, E.J. Herup, L.N.B. Gummadi, Finite element analysis of low-velocity impact on composite sandwich plates. Composite Structures, 49, 209-227, 2000.

[13] T. Besant, G.A.O. Davies, D. Hitchings, Finite element modelling of low velocity impact of composite sandwich panels. Composites Part A, 32, 1189-1196, 2001. 
[14] G.A.O. Davies, D. Hitchings, T. Besant, A. Clarke, C. Morgan. Compression after impact strength of composite sandwich panels. Composite Structures, 63, 1-9, 2004.

[15] L. Kärger, J. Baaran and J. Teßmer, Rapid Simulation of Impacts on Composite Sandwich Panels Inducing Barely Visible Damage. Composite Structures, in press, 2006.

[16] R. Rolfes, J. Baaran, J. Juhasz, A. Kling, Ph. Nolden and G. Kuhlmann, High Performance Tools for Failure, Damage Tolerance and Stability Analysis of Composite Structures. Proceedings WCCM V, Vienna, 2002.

[17] J. Baaran, L. Kärger and A. Wetzel, CODAC Version 1.6 Manual. DLR, Institute FA, Braunschweig, Internal Report IB 131-2005/38, 2005.

[18] L. Kärger, A. Wetzel, R. Rolfes and K. Rohwer, A three-layered sandwich element with improved transverse shear stiffness and stresses based on FSDT. Computers and Structures, in press, 2006.

[19] R. Rolfes and K. Rohwer, Improved transverse shear stresses in composite finite elements based on first order shear deformation theory. International Journal for Numerical Methods in Engineering, 40, 51-60, 1997.

[20] A. Wetzel, L. Kärger, R. Rolfes and K. Rohwer, Evaluation of two finite element formulations for a rapid 3D stress analysis of sandwich structures. Computers and Structures, 83, 1537-1545, 2005.

[21] R. Rolfes, K. Rohwer and M. Ballerstaedt, Efficient Linear Transverse Normal Stress Analysis of Layered Composite Plates. Computers and Structures, 68, 643-652, 1998.

[22] N.J. Pagano, Exact solutions for composite laminates in cylindrical bending. Journal of Composite Materials, 3, 398-411, 1969.

[23] N.J. Pagano, Exact solutions for rectangular bidirectional composites and sandwich plates. Journal of Composite Materials, 4, 20-34, 1970.

[24] K. Wolf, F. Hähnel and K. Steinbach, Internal communications. Department of Aerospace Technology, Dresden University of Technology.

[25] Z. Hashin, Failure Criteria for Unidirectional Fibre Composites. Journal of Applied Mechanics, 47, 329-334, 1980.

[26] F.-K. Chang, K.-Y Chang, A progressive damage model for laminated composites containing stress concentrations. Journal of Composite Materials, 21, 834-855, 1987.

[27] R. Olsson, Improved theory for contact indentation of sandwich panels. AIAA Journal, 6 , 1238-1244, 1996. 